\title{
Enforced expression of miR-101 enhances cisplatin sensitivity in human bladder cancer cells by modulating the cyclooxygenase- 2 pathway
}

\author{
QIANG BU ${ }^{1}$, YUE FANG ${ }^{2}$, YUAN CAO ${ }^{2}$, QIAOYUN CHEN ${ }^{2}$ and YANGCHEN LIU ${ }^{3}$ \\ ${ }^{1}$ Department of Urology, Danyang People's Hospital, Zhenjiang; ${ }^{2}$ Department of Central Laboratory, \\ The Affiliated People's Hospital, Jiangsu University, Zhenjiang; ${ }^{3}$ Department of Oncology, \\ Taixing People's Hospital, Taixing, Jiangsu, P.R. China
}

Received October 28, 2013; Accepted June 9, 2014

DOI: $10.3892 / \mathrm{mmr} .2014 .2455$

\begin{abstract}
Alterations in microRNA (miRNA) expression have been shown to be involved in the tumor response to chemotherapy. However, the possible role of miR-101 in cisplatin sensitivity in human bladder cancer cells remains unclear. In this study, quantitative polymerase chain reaction and western blotting were utilized to determine the expression profiles of miR-101 and cyclooxygenase-2 (COX-2) in human bladder cancer cells. The effect of miR-101 and small interfering RNA (siRNA) against COX-2 on cell viability was evaluated using MTT assays, and apoptosis levels were determined using fluorescence-activated cell sorting analysis of Annexin V/propidium iodide-stained cells. Luciferase reporter plasmids were constructed to confirm direct targeting. This study found that the expression of miR-101 was downregulated in the cisplatin-resistant cell line T24/CDDP as compared with that in the parental line, T24. Furthermore, overexpression of miR-101 significantly increased the anti-proliferative effects and apoptosis induced by cisplatin, whereas knockdown of miR-101 significantly decreased the anti-proliferative effects and apoptosis induced by cisplatin. In addition, downregulation of miR-101 induced cell survival and cisplatin resistance through the upregulation of COX-2 expression. Luciferase gene reporter assays confirmed that COX-2 was a direct target gene of miR-101. Inhibition of COX-2 using COX-2 siRNA abrogated the cisplatin resistance induced by miR-101 downregulation. These results suggest that miR-101 may provide a novel mechanism for understanding cisplatin resistance in bladder cancer by modulating the COX-2 pathway.
\end{abstract}

Correspondence to: Dr Yangchen Liu, Department of Oncology, Taixing People's Hospital, No. 1 Changzheng Road, Taixing, Jiangsu, P.R. China

E-mail: 1yc201308@sina.cn

Key words: miR-101, cyclooxygenase-2, bladder cancer

\section{Introduction}

Human bladder cancer is the fourth most common malignancy in males, and the 10th most common malignancy in females (1). There are currently numerous therapeutic techniques available for the treatment of bladder cancer, including intravesical chemotherapy, surgery, radiation therapy and systemic chemotherapy, which are selected depending on the extent of the disease $(2,3)$. Despite these various treatment options, patients with advanced bladder cancer have a five-year survival rate of 20-40\% (4). For patients with advanced bladder cancer, cisplatin-based combination chemotherapy, for example methotrexate, vinblastine, doxorubicin and cisplatin or gemcitabine and cisplatin, is the current choice of treatment. However, one of the most significant limitations in the efficacy of cisplatin-based combination chemotherapy is that numerous tumors either demonstrate an inherent resistance to the chemotherapy or acquire resistance following an initial response (5). The molecular mechanisms underlying this chemoresistance have yet to be elucidated, although cisplatin resistance has been associated with a reduction in the apoptotic response of certain types of cancer cells (6). There is therefore a requirement to develop novel drugs that are able to reverse the resistance to chemotherapy and enhance sensitivity to platinum-based chemotherapy drugs.

microRNAs (miRNAs) are endogenous, non-coding RNA molecules of 19-25 nucleotides in length. The pairing of miRNAs with target mRNAs carrying a partially complementary sequence in the $3^{\prime}$ untranslated region (UTR) results in the translational repression and/or degradation of the mRNA and thus the silencing of associated genes (7-9). Alterations in the expression patterns of miRNAs that regulate genes involved in cell proliferation, differentiation or apoptosis have been found in various human tumors (10-12). Therefore, miRNA expression profiles may be utilized to determine the expected sensitivity of tumors to chemotherapy prior to treatment, and changes in the expression of miRNAs during treatment could act as markers for the response of tumors to the chemotherapy. In a comparison of doxorubicin-resistant and -sensitive MCF-7 breast cancer cell lines, Kovalchuk et al (13) identified 137 deregulated miRNAs, while 103 deregulated miRNAs were identified in a comparison of cisplatin-resistant and -sensitive MCF-7 cells (13). In addition, 
Nordentoft et al (14) found that the expression of 15 miRNAs correlated with chemotherapy response and that the expression of five miRNAs correlated with survival time in patients with bladder cancer. It was further found that a reduction in miR-138 expression resulted in an increase in cisplatin sensitivity, while an upregulation of miR-27a and miR-642 expression also increased cisplatin sensitivity (14).

Increasing evidence has indicated that there is an association between chronic inflammation and tumorigenesis. Cyclooxygenase-2 (COX-2) is a key regulator of inflammation-producing prostaglandins and thus could be involved in inflammation-associated cancer development (15). Increased COX-2 expression has been found in a number of apoptosis-resistant cancer cells (16); therefore, COX-2 may be a potential target in the reversal of this apoptosis resistance. The expression of COX-2 has been shown to be modulated by several mechanisms, including the action of miRNA (17). He et al (18) reported that miR-101 downregulation in gastric cancer correlated with the overexpression of COX-2 and tumor growth. miR-101 may therefore influence cisplatin sensitivity in cancer cells through the inhibition of COX-2 expression.

To the best of our knowledge, the association between miR-101 and cisplatin resistance in bladder cancer cells has not been reported. In the present study, we explored the association between cisplatin sensitivity and miR-101 in bladder cancer.

\section{Materials and methods}

Cell culture. T24 human urinary bladder transitional cell carcinoma cells were cultured in RPMI-1640 medium supplemented with $10 \%$ heat-inactivated fetal bovine serum (FBS) (Sigma-Aldrich, St. Louis, MO, USA) and 1\% penicillin/streptomycin sulfate (Beyotime Institute of Biotechnology, Haimen, China) under an atmosphere of $95 \%$ air and $5 \% \mathrm{CO}_{2}$ at $37^{\circ} \mathrm{C}$. T24 human urinary bladder transitional cell carcinoma cells were purchased from the Shanghai Institute of Biochemistry and Cell Biology (Shanghai, China). The cisplatin-resistant T24 cell line (T24/CDDP) was established as described previously (6).

Transfection of miRNA mimics and inhibitors. T24/CDDP and T2 4 cells were plated in six-well plates $\left(5 \times 10^{5}\right.$ cells/well). A total of $100 \mathrm{nM}$ miR-101 mimic or $100 \mathrm{nM}$ miRNA mimic control was transfected in T24/CDDP cells, and $100 \mathrm{nM}$ miR-101 inhibitor or $100 \mathrm{nM}$ miRNA inhibitor control was transfected in T24 cells, using Lipofectamine ${ }^{\mathrm{TM}} 2000$ (Invitrogen Life Technologies, Carlsbad, CA, USA) according to the manufacturer's instructions. The sequences were as follows: miR-101 mimic, 5'-UACAGUACUGUGAUAACUGAA-3'; miR-101 inhibitor, 5'-CUUCAGUUAUCACAGUACUGUA-3'; and negative control, 5'-GUGGAUAUUGUUGCCAUCA-3'.

Quantitative polymerase chain reaction ( $q P C R$ ) for miR-101. Total RNA from cells was extracted using TRIzol $^{\mathrm{TM}}$ reagent (Invitrogen Life Technologies). Levels of the mature forms of miR-101 in the cells were determined using a TaqMan ${ }^{\circledR}$ miRNA assay kit (Applied Biosystems, Foster City, CA, USA), and data were normalized to U6 expression (Applied Biosystems). The qPCR procedure was performed according to the manufacturer's instructions. The fold change for miR-101 was calculated using the $2^{-\Delta \Delta C t}$ method (19).

Cell viability assay. Cells were seeded into 96-well plates in RPMI-1640 medium containing 10\% FBS. Following $24 \mathrm{~h}$ in culture, the cells were treated with serial dilutions of cisplatin. Approximately $72 \mathrm{~h}$ after cisplatin treatment, MTT was added to a final concentration of $0.5 \mathrm{mg} / \mathrm{ml}$ and the cells were then incubated for $4 \mathrm{~h}$ at $37^{\circ} \mathrm{C}$. The optical density was read at $490 \mathrm{~nm}$ with a microplate spectrophotometer (iMark; Bio-Rad, Hercules, CA, USA). Each experiment was performed in triplicate and repeated three times.

Western blot analysis. T24 or T24/CDDP cells were plated in six-well plates $\left(5 \times 10^{5}\right.$ cells/well $)$ and, $72 \mathrm{~h}$ after transfection with miR-101 mimic or inhibitors, the cells were harvested and homogenized with lysis buffer. Total protein was separated by denaturing $10 \%$ SDS-PAGE. Western blot analysis was performed as described previously (20). The primary antibodies against COX-2 and GAPDH were purchased from Cell Signaling Technology, Inc., (Danvers, MA, USA) and Santa Cruz Biotechnology, Inc., (Santa Cruz, CA, USA), respectively. Protein levels were normalized to GAPDH and the subsequent fold changes were determined.

Apoptosis detection. Apoptosis was assessed by Annexin V/propidium iodide (PI) staining $48 \mathrm{~h}$ after treatment using flow cytometry (FACSCalibur ${ }^{\mathrm{TM}}$; BD Biosciences, Heidelberg, Germany), according to the manufacturer's instructions. The percentage of early apoptotic cells was determined by the quantitative analysis of Annexin V-fluorescein isothiocyanate-positive/PI-negative plots using the CellQuest software (BD Biosciences).

Luciferase assay. The luciferase reporter plasmids were constructed as described previously (21). A fragment of the 3'UTR COX-2 mRNA, which carried a putative miR-101 complementary site (NM_000963; 3'-UTR, 1,735-1,741), and a mutant variant were cloned into a pGL3 vector (pGL3-empty; Promega Corporation, Madison, WI, USA) with a downstream firefly luciferase gene ( $\mathrm{XbaI}$ site) to obtain luciferase reporter constructs (pGL3-COX2-wildtype and pGL3-COX2-mutant, respectively). The target gene was predicted by TargetScan Human 6.2 (http://www.targetscan.org) as described previously (22). Cells were seeded in 24-well plates and co-transfected with miR-101 mimics and either pGL3-COX2-wildtype or pGL3-COX2-mutant.

siRNA transfection. siRNA against COX-2 (5'-AACTGCTCAACACCGGAATTT-3') and negative control was purchased from Cell Signaling Technology, Inc., and the transfection was performed according to the manufacturer's instructions. The transfection efficiency was evaluated using fluorescence microscopy by calculating the percentage of fluorescein-labeled cells. Cells were lysed in lysis buffer $48 \mathrm{~h}$ after transfection for western blot analyses.

Nude mouse xenografts. Nude mouse xenografts were performed as previously described (23). Nude mice were purchased from Vital River Laboratories (Beijing, China) and 
maintained at the Experimental Animal Center of Jiangsu University (Jiangsu, China). Each mouse was subcutaneously inoculated with $2 \times 10^{6}$ T24/CDDP cells, which had been transfected with miR-101 or miR-control, in $0.2 \mathrm{ml}$ medium in the forelimb. Tumor sizes were measured every three days in two dimensions using a caliper and the volume $(\mathrm{V})\left(\mathrm{mm}^{3}\right)$ was calculated using the following formula: $\mathrm{V}=0.5 \mathrm{x}$ larger diameter $\mathrm{x}$ (smaller diameter) ${ }^{2}$. The mice were sacrificed and the tumors were excised and weighed 21 days after the initial inoculation. All procedures involving animals were approved by the Animal Care and Use Committee of Jiangsu University (Zhenjiang, China).

Statistical analysis. Quantitative data are presented as the mean \pm standard deviation. The Student's t-test was used to determine statistical significance. $\mathrm{P}<0.05$ was considered to indicate a statistically significant difference.

\section{Results}

miR-101 is downregulated in T24/CDDP cells as compared with T24 cells. The doses of cisplatin required for a $50 \%$ inhibition of T24 and T24/CDDP cells were 5.2 and $14.7 \mu \mathrm{g} / \mathrm{ml}$, respectively (Fig. 1). The resistance to cisplatin in the T24/CDDP cells was 2.83 -fold greater than that in the parent T24 cells. qPCR for miR-101 expression further verified that miR-101 was significantly downregulated in T24/CDDP cells, with an expression level of $0.27 \pm 0.05$ relative to that in the parental cells.

miR-101 modulates the sensitivity of cisplatin in T24 cells. To directly assess the association between miR-101 and cisplatin resistance in the T24 cells, the expression of miR-101 was further downregulated in the T24 cell line. The miR-101 inhibitor effectively reduced the expression of miR-101 $(\mathrm{P}<0.05)$ (Fig 2A). T24 cells transfected with the miR-101 inhibitor exhibited significantly enhanced resistance to cisplatin compared with the negative control transfected cells $(\mathrm{P}<0.05)$ (Fig. 2B). This suggests that decreasing miR-101 expression contributes to cisplatin resistance in T24 cells. The miR-101 mimic significantly induced the expression of miR-101 in T24/CDDP cells $(\mathrm{P}<0.05)$ (Fig. 2C), and MTT assay revealed that T24/CDDP cells transfected with the miR-101 mimics exhibited significantly decreased resistance to cisplatin compared with the negative control-transfected cells (Fig. 2D). Tumor growth curves were generated by measuring the size of tumors in nude mice over a period of 21 days. Following T24/CDDP cell injection, the volume of the tumors in the miR-101 mimic and negative control groups showed differential progression (Fig. 2E). Additionally, the tumors in the T24/CDDP plus miR-101 mimic group were significantly lighter than those in the negative control group $(\mathrm{P}<0.05)$ (Fig. $2 \mathrm{~F})$. These results suggest that miR-101 regulates cisplatin resistance in the T24 human bladder cancer cell line.

COX-2 is a directtarget gene ofmiR-101. TargetScan Human 6.2 (http://www.targetscan.org) predicted that COX-2 was a target gene of the miR-101 (Fig. 3A). A luciferase reporter vector with the putative COX-2 3' UTR target site for the miR-101 downstream of the luciferase gene (pGL3-COX2-wildtype) was therefore constructed in order to test this in vitro. The
$\mathbf{A}$

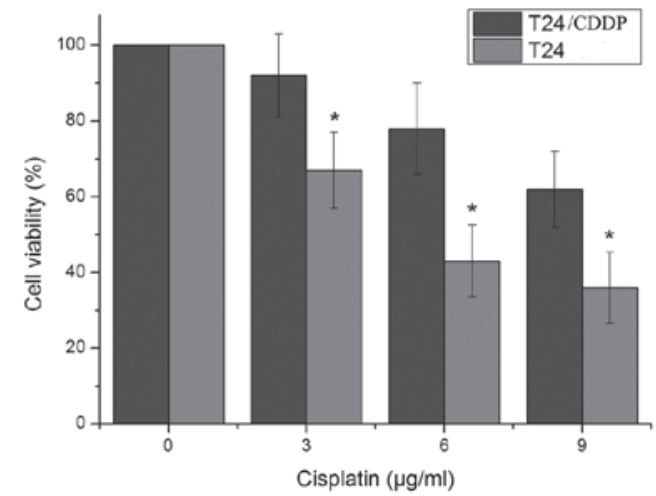

B

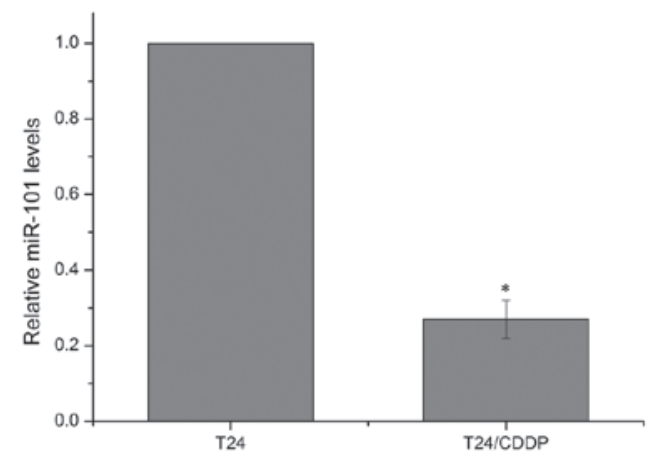

Figure 1. Expression of miR-101 in bladder cancer cell lines. (A) The sensitivity of T24 and T24/CDDP cells to cisplatin was determined by MTT assay. (B) miR-101 levels in the T24 and T24/CDDP cells were measured by quantitative polymerase chain reaction. ${ }^{*} \mathrm{P}<0.05$ vs. the $\mathrm{T} 24$ cells. miR-101, microRNA-101.

luciferase reporter vector, together with the miR-101 mimic or the negative control, was transfected into T24/CDDP cells. A significant decrease in relative luciferase activity was noted when pGL3-COX2-wildtype was co-transfected with the miR-101 mimics into T24/CDDP cells. However, the co-transfection of the pGL3-COX2-mutant with the miR-101 mimic did not affect reporter activity $(\mathrm{P}>0.05)$ (Fig. 3B). Western blot assay revealed that T24/CDDP cells transfected with miR-101 mimic exhibited significantly decreased protein levels of COX-2 as compared with the cells transfected with the negative control (Fig. 3C and D). These results suggest that COX-2 is a target gene of miR-101.

COX-2 is involved in cisplatin resistance in T24/CDDP cells. Previous studies have shown that COX-2 is involved in drug resistance in various types of cancer $(24,25)$; however, its role in cisplatin sensitivity in the T24/CDDP cell line remains unclear. To explore the association between COX-2 and cisplatin-induced cytotoxicity, COX-2 siRNA or a negative control was transfected into T24/CDDP cells, followed by treatment with various doses of cisplatin. COX-2 siRNA effectively reduced the COX-2 protein level (Fig. 4A and B). Furthermore, T24/CDDP cells that were treated with COX-2 siRNA had decreased survival compared with the control group (Fig. 4C). Of note, the reduced survival rates exhibited by the COX-2 siRNA-treated T24/CDDP cells were similar to those exhibited by the cells with miR-101 overexpression. This suggests that miR-101 reversed cisplatin resistance via the regulation of COX-2 in the T24/CDDP cells. 
A

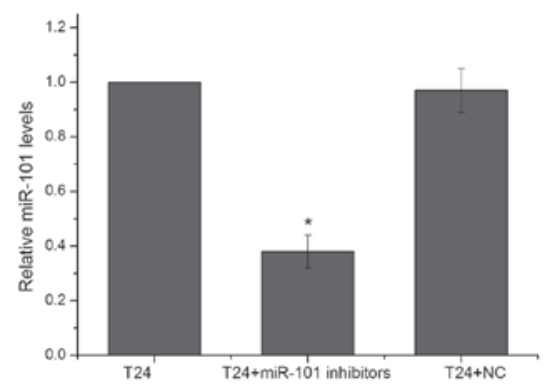

C

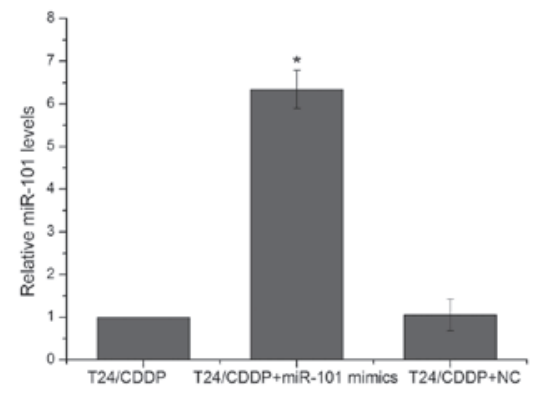

$\mathbf{E}$

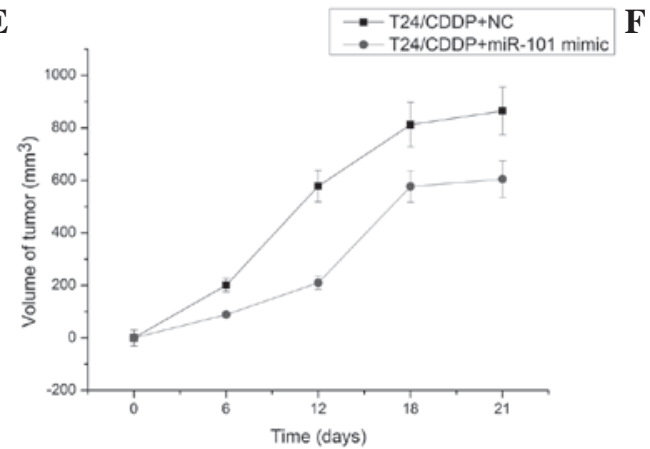

B

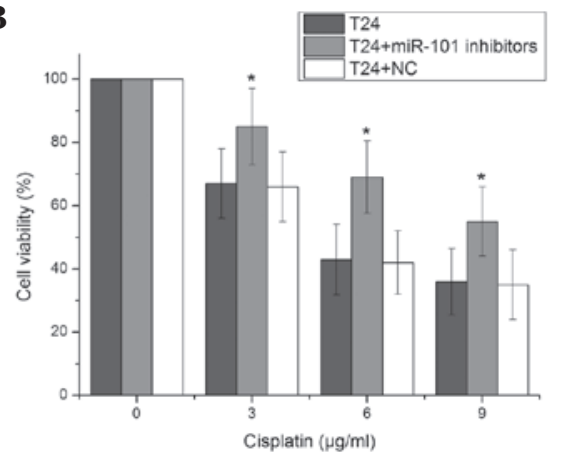

D

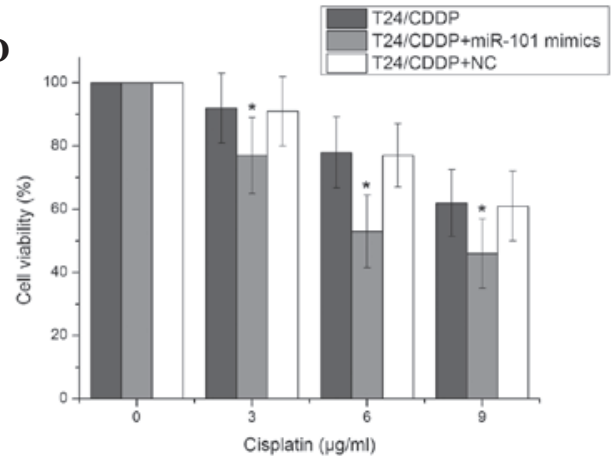

F

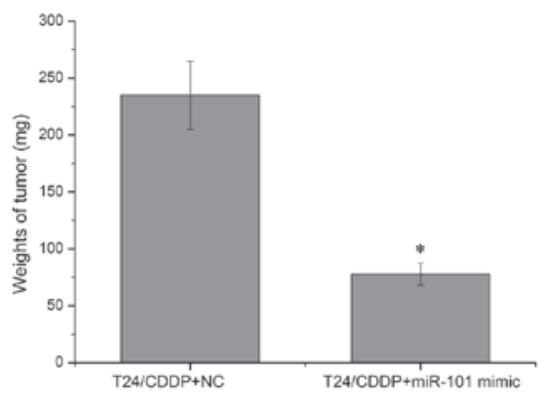

Figure 2. miR-101 modulates the sensitivity of cisplatin in T24 cells. (A) Levels of miR-101 expression in T24 cells measured by qPCR. (B) The cell viability in T24 cells was determined by MTT assay. (C) Levels of miR-101 in T24/CDDP cells measured by qPCR. (D) The cell viability in T24/CDDP cells was determined by MTT assay. (E) Tumor growth curves. (F) Tumor mass in the T24/CDDP+NC and T24/CDDP+miR-101 mimic groups. "P<0.05 vs. the NC group. NC, negative control; miR-101, microRNA-101; qPCR, quantitative polymerase chain reaction.

A

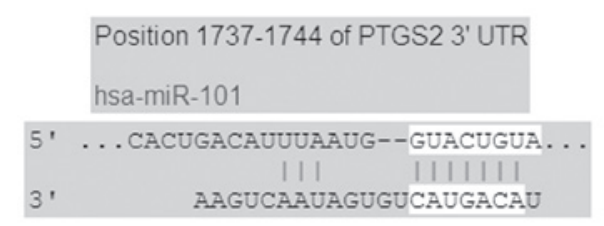

C

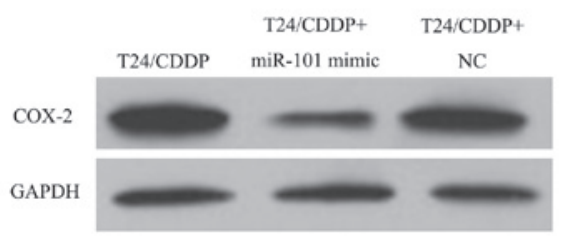

B

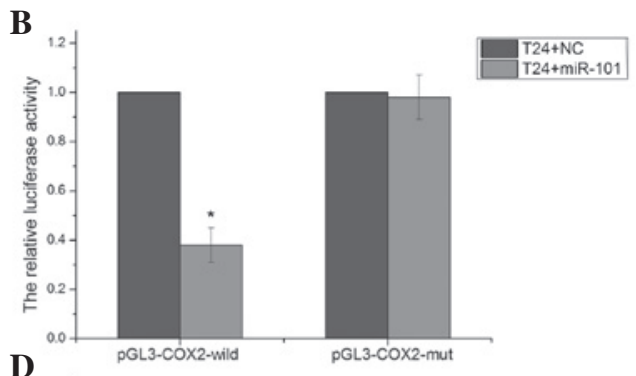

D

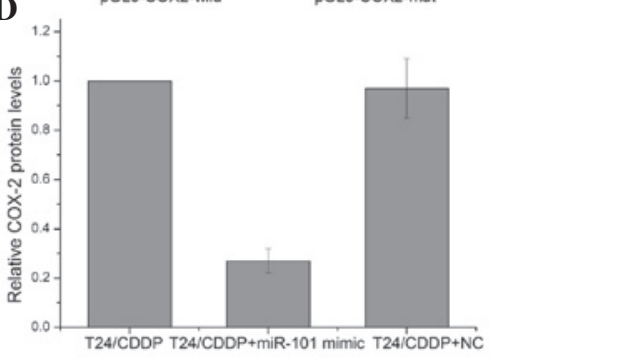

Figure 3. COX-2 is predicted to be the target gene of miR-101 following dual luciferase assay in T24/CDDP cells. (A) TargetScan predicted that COX-2 was the target gene of the miR-101. (B) A significant decrease in the relative luciferase activity was observed following co-transfection of pGL3-BCL2-3'-UTR with the miR-101 mimic. (C and D) miR-101 modulated the expression of COX-2 in T24/CDDP cells. "P<0.05 vs. the NC group. NC, negative control; COX-2, cyclooxygenase-2; miR-101, microRNA-101. 
A

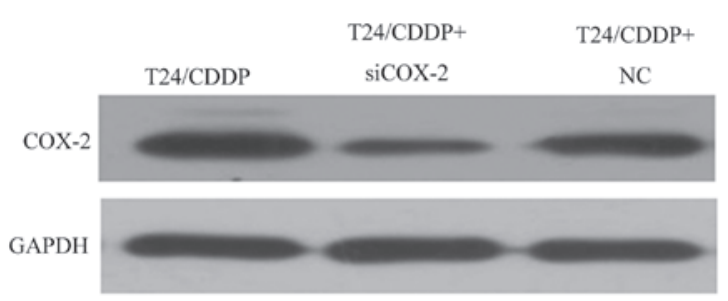

B

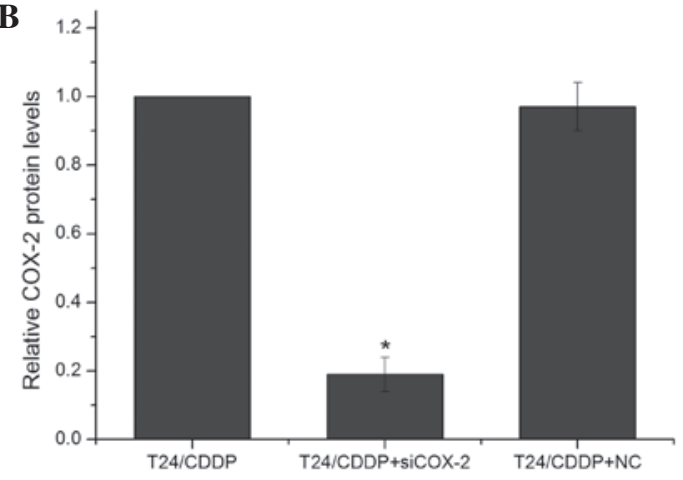

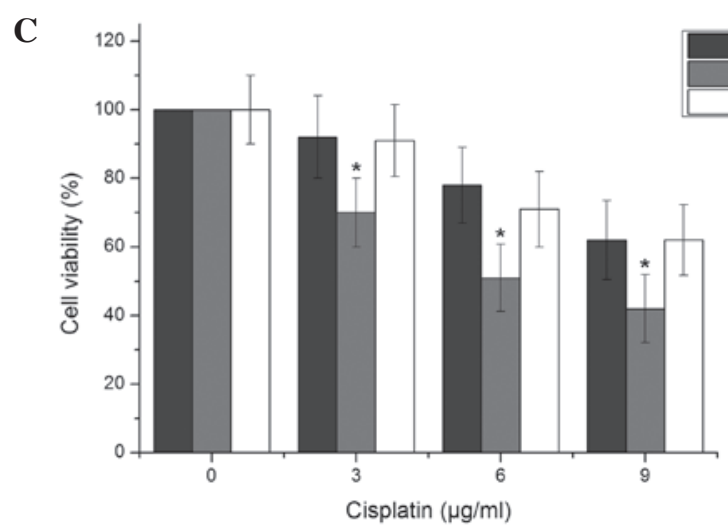

Figure 4. COX-2 is involved in cisplatin resistance in T24/CDDP cells. (A and B) COX-2 siRNA effectively reduced the expression of COX-2 in T24/CDDP cells. (C) COX-2 siRNA decreased the survival rate in T24/CDDP cells. " $\mathrm{P}<0.05$ vs. the NC group. NC, negative control; COX-2, cyclooxygenase-2; siRNA, small interfering RNA.

A

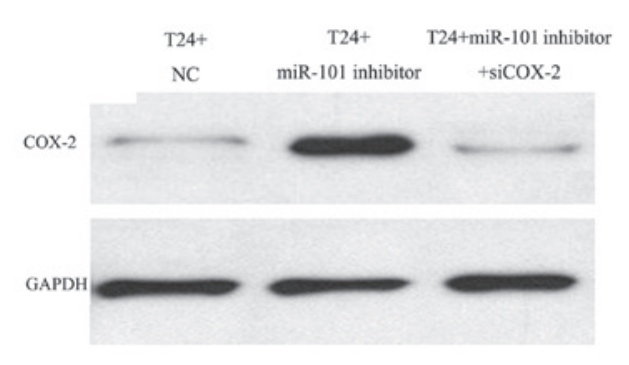

D

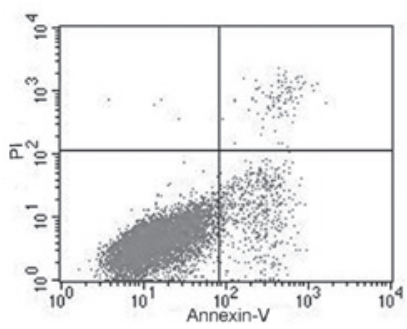

B

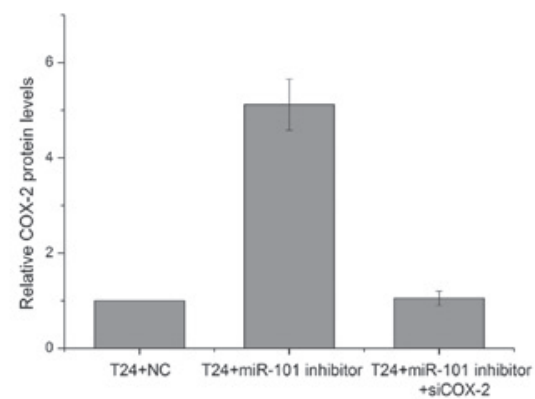

$\mathbf{E}$

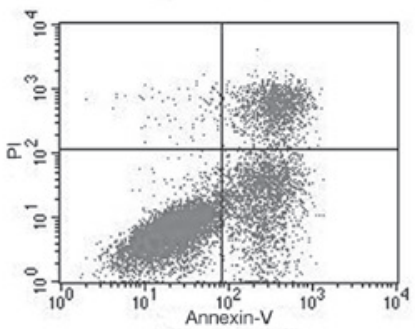

C

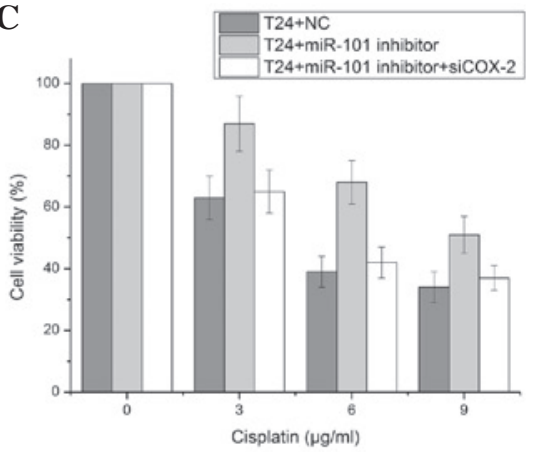

$\mathbf{F}$

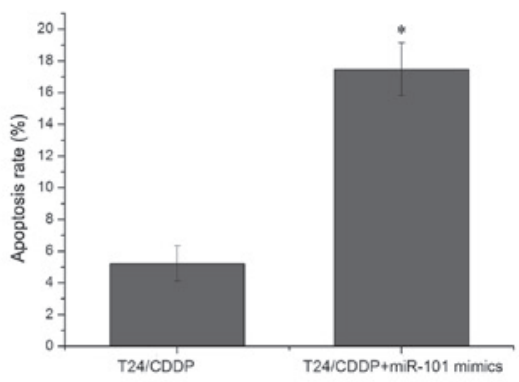

Figure 5. miR-101 regulates cisplatin sensitivity in T24 cells by targeting COX-2. (A and B) miR-101 inhibitors increased the expression of COX-2 protein in T24 cells, which could be partly alleviated by the COX-2 siRNA. (C) T24 cells transfected with miR-101 inhibitor exhibited greatly enhanced cisplatin resistance compared with the NC-transfected cells, which could be partly alleviated by the COX-2 siRNA. (D-F) The T24/CDDP cells transfected with miR-101 mimics exhibited a higher apoptosis rate than the NC group. "P<0.05 vs. the NC group; NC, negative control; COX-2, cyclooxygenase-2; siRNA, small interfering RNA; PI, propidium iodide.

miR-101 regulates cisplatin sensitivity in $T 24$ cells by targeting $C O X-2$. It was hypothesized in this study that miR-101 modulates the cisplatin resistance of bladder cancer cells by repressing COX-2 protein expression. To ascertain this, miR-101 inhibitor or negative control was transfected into T24 cells for the detection of changes in COX-2 expression levels. 
Western blot analysis demonstrated significantly increased COX-2 protein levels in the miR-101 mimic-transfected T24 cells as compared with the negative control-transfected T24 cells $72 \mathrm{~h}$ after transfection,. This was observed to be in part alleviated by transfection with COX-2 siRNA (Fig. 5A and B). Enhanced cell viability was observed in the T24 cells transfected with miR-101 inhibitor as compared with negative control-transfected cells, which could be partly alleviated by the COX-2 siRNA (Fig. 5C). miR-101 may therefore play a role in the development of cisplatin resistance, in part through the modulation of apoptosis. To confirm this hypothesis, cisplatin-induced apoptosis was evaluated in T24/CDDP cells following transfection with the miR-101 mimic or the negative control. A marked increase in apoptosis, as assessed by flow cytometry, was observed in the T24/CDDP cells transfected with the miR-101 mimic following cisplatin treatment, as compared with the negative control-transfected cells (Fig. 5D-F). These results showed that miR-101 modulates the cisplatin resistance of T24/CDDP cells, and this may be through the inhibition of COX-2 protein expression.

\section{Discussion}

Resistance to chemotherapeutic agents is a critical issue in cancer treatment, including the treatment of bladder cancer. This resistance may result from the failure of the apoptosis that is activated in response to drug treatment. Combined chemotherapy using cisplatin resulted in a response rate of $60-80 \%$ in patients with advanced bladder cancer, but the subsequent emergence of chemoresistance meant that complete remission was sustained in only $15 \%$ of affected patients (26). An enhanced understanding of the mechanisms contributing to cisplatin resistance may facilitate predictions of the clinical response to therapy. The aim of this study was to gain insight into the pathway leading to cisplatin-induced apoptosis in human bladder cancer cells. This study showed for the first time that cisplatin-induced drug resistance was associated with the downregulation of miR-101 in the T24 bladder cancer cell line. miR-101 may regulate the survival of T24 cells by modulating COX-2 expression.

Overexpression of miR-101 has been found to suppress cell proliferation and impair invasive potential in prostate cancer, bladder carcinoma and colon cancer (27-29). Additionally, Zhang et al (30) demonstrated that the downregulation of miR-101 in non-small cell lung cancer acted as a tumor promoter by stimulating cell proliferation and invasion and inhibiting paclitaxel-induced apoptosis. In an in vitro study of gastric cancer, Wang et al (31) showed that gastric cancer cell proliferation, migration and invasion could be inhibited by the ectopic expression of miR-101. Furthermore, Chen et al found that ectopic miR-101 acted to sensitize human tumor cells to radiation via the inhibition of DNA repair by targeting ataxia telangiectasia mutated and DNA-dependent protein kinase catalytic subunits (32). The present study found that miR-101 expression was significantly downregulated in cisplatin-resistant bladder cancer cells as compared with that in parent cells. This observed downregulation of miR-101 may be involved in the occurrence of cisplatin resistance. The biological functions of miR-101 in bladder cancer were further explored. Expression of miR-101 in cisplatin-resistant bladder cancer cells inhibited cell proliferation and promoted apoptosis. The identified role of miR-101 in bladder cancer cells from this study was consistent with that observed in other cancers (33). Taken together, these results further confirm that miR-101 functions as a tumor suppressor.

Following TargetScan software analysis, it was predicted that COX-2 could be a direct target of miR-101 due to a seed region within miR-101, which is able to bind to the COX-2 mRNA 3'-UTR. A luciferase reporter vector (pGL3-COX2-wildtype) containing the 3'-UTR of COX-2 with a putative miR-101 complementary region was constructed. A significantly lower level of luciferase activity was detected when T24/CDDP cells were co-transfected with the miR-101 mimic and pGL3-COX2-wildtype, indicating a direct interaction between miR-101 and COX-2 mRNA. It has been suggested that COX-2 inhibitors can sensitize drug-resistant tumor cells to chemotherapy (34). Nonsteroidal anti-inflammatory drugs are often used for the treatment of colon cancer, either alone or in combination with other chemotherapeutic agents. Combining flurbiprofen, sulindac or indomethacin with 5-fluorouracil resulted in an enhancement of cytotoxicity (35). In this study, the re-expression of miR-101 in cisplatin-resistant bladder cancer cells decreased COX-2 expression, inhibited cell proliferation and promoted apoptosis. The results of the siRNA assays confirmed that COX-2 could modulate cisplatin sensitivity in bladder cancer cells. Knockdown of COX-2 significantly decreased cell survival with an overall effect that was similar to that of miR-101 overexpression.

In conclusion, this study has presented the first evidence, to the best of our knowledge, that miR-101 may be involved in the development of cisplatin resistance in human bladder cancer cell lines. miR-101 was shown to modulate the resistance of bladder cell lines to cisplatin, at least in part, through targeting COX-2 expression. Therapeutic strategies targeting drug resistance associated with miRNAs, such as hsa-miR-101, may be another promising way to enhance therapeutic effects.

\section{Acknowledgements}

This study was supported by funding from the Science and Technology Support Plan of Zhenjiang City (FZ2012003).

\section{References}

1. Bo J, Yang G, Huo K, et al: microRNA-203 suppresses bladder cancer development by repressing bcl-w expression. FEBS J 278: 786-792, 2011.

2. Donat SM: Integrating perioperative chemotherapy into the treatment of muscle-invasive bladder cancer: strategy versus reality. J Natl Compr Canc Netw 7: 40-47, 2009.

3. Kakizoe T, Mucci LA, Albertsen PC and Droller MJ: Screening for bladder cancer: theoretical and practical issues in considering the treated and untreated natural history of the various forms of the disease. Scand J Urol Nephrol Suppl: 191-212, 2008.

4. Noguchi S, Mori T, Hoshino Y, et al: MicroRNA-143 functions as a tumor suppressor in human bladder cancer T24 cells. Cancer Lett 307: 211-220, 2011.

5. Andrews PA and Howell SB: Cellular pharmacology of cisplatin: perspectives on mechanisms of acquired resistance. Cancer Cells 2: 35-43, 1990.

6. Cho HJ, Kim JK, Kim KD, et al: Upregulation of Bcl-2 is associated with cisplatin-resistance via inhibition of Bax translocation in human bladder cancer cells. Cancer Lett 237: 56-66, 2006.

7. Bartel DP: MicroRNAs: genomics, biogenesis, mechanism, and function. Cell 116: 281-297, 2004. 
8. Kim VN and Nam JW: Genomics of microRNA. Trends Genet 22: 165-173, 2006.

9. Shyu AB, Wilkinson MF and van Hoof A: Messenger RNA regulation: to translate or to degrade. EMBO J 27: 471-481, 2008

10. Iorio MV, Ferracin M, Liu CG, et al: MicroRNA gene expression deregulation in human breast cancer. Cancer Res 65 7065-7070, 2005.

11. Lu J, Getz G, Miska EA, et al: MicroRNA expression profiles classify human cancers. Nature 435: 834-838, 2005.

12. Volinia S, Calin GA, Liu CG, et al: A microRNA expression signature of human solid tumors defines cancer gene targets. Proc Natl Acad Sci USA 103: 2257-2261, 2006.

13. Kovalchuk O, Filkowski J, Meservy J, et al: Involvement of microRNA-451 in resistance of the MCF-7 breast cancer cells to chemotherapeutic drug doxorubicin. Mol Cancer Ther 7 : 2152-2159, 2008.

14. Nordentoft I, Birkenkamp-Demtroder K, Agerbæk M, et al: miRNAs associated with chemo-sensitivity in cell lines and in advanced bladder cancer. BMC Med Genomics 5: 40, 2012.

15. Greenhough A, Smartt HJ, Moore AE, et al: The COX-2/PGE2 pathway: key roles in the hallmarks of cancer and adaptation to the tumour microenvironment. Carcinogenesis 30: 377-386, 2009

16. Hasegawa $\mathrm{K}$, Ishikawa $\mathrm{K}$, Kawai $\mathrm{S}$, et al: Overcoming paclitaxel resistance in uterine endometrial cancer using a COX-2 inhibitor. Oncol Rep 30: 2937-2944, 2013.

17. Wu XL, Cheng B, Li PY, et al: MicroRNA-143 suppresses gastric cancer cell growth and induces apoptosis by targeting COX-2. World J Gastroenterol 19: 7758-7765, 2013.

18. He XP, Shao Y, Li XL, et al: Downregulation of miR-101 in gastric cancer correlates with cyclooxygenase-2 overexpression and tumor growth. FEBS J 279: 4201-4212, 2012.

19. Livak KJ and Schmittgen TD: Analysis of relative gene expression data using real-time quantitative PCR and the 2(-Delta Delta C(T)) Method. Methods 25: 402-408, 2001.

20. Xia L, Zhang D, Du R, et al: miR-15b and miR-16 modulate multidrug resistance by targeting BCL2 in human gastric cancer cells. Int J Cancer. 123: 372-379, 2008.

21. Srivastava SK, Tetsuka T, Daphna-Iken D and Morrison AR: IL-1 beta stabilizes COX II mRNA in renal mesangial cells: role of 3'-untranslated region. Am J Physiol 267: F504-F508, 1994.

22. Xiang KM and Li XR: MiR-133b acts as a tumor suppressor and negatively regulates TBPL1 in colorectal cancer cells. Asian Pac J Cancer Prev 15: 3767-3772, 2014.
23. Xu T, Zhou Q, Zhou J, et al: Carboxyl terminus of Hsp70-interacting protein (CHIP) contributes to human glioma oncogenesis. Cancer Sci 102: 959-966, 2011.

24. Kang JH, Song KH, Jeong KC, et al: Involvement of Cox-2 in the metastatic potential of chemotherapy-resistant breast cancer cells. BMC Cancer 11: 334, 2011.

25. Akutsu Y, Hanari N, Yusup G, et al: COX2 expression predicts resistance to chemoradiotherapy in esophageal squamous cell carcinoma. Ann Surg Oncol 18: 2946-2951, 2011.

26. Hong JH, Lee E, Hong J, Shin YJ and Ahn H: Antisense Bcl2 oligonucleotide in cisplatin-resistant bladder cancer cell lines. BJU Int 90: 113-117, 2002.

27. Friedman JM, Liang G, Liu CC, et al: The putative tumor suppressor microRNA-101 modulates the cancer epigenome by repressing the polycomb group protein EZH2. Cancer Res 69: 2623-2629, 2009

28. Strillacci A, Griffoni C, Sansone P, et al: MiR-101 downregulation is involved in cyclooxygenase-2 overexpression in human colon cancer cells. Exp Cell Res 315: 1439-1447, 2009.

29. Varambally S, Cao Q, Mani RS, et al: Genomic loss of microRNA-101 leads to overexpression of histone methyltransferase EZH2 in cancer. Science 322: 1695-1699, 2008.

30. Zhang JG, Guo JF, Liu DL, Liu Q and Wang JJ: MicroRNA-10 exerts tumor-suppressive functions in non-small cell lung cancer through directly targeting enhancer of zeste homolog 2 . J Thorac Oncol 6: 671-678, 2011.

31. Wang HJ, Ruan HJ,He XJ, et al: MicroRNA-101 is down-regulated in gastric cancer and involved in cell migration and invasion. Eur J Cancer 46: 2295-2303, 2010.

32. Chen S, Wang H, Ng WL, Curran WJ and Wang Y: Radiosensitizing effects of ectopic miR-101 on non-small-cell lung cancer cells depend on the endogenous miR-101 level. Int J Radiat Oncol Biol Phys 81: 1524-1529, 2011.

33. Xu Y, An Y, Wang Y et al: miR-101 inhibits autophagy and enhances cisplatin-induced apoptosis in hepatocellular carcinoma cells. Oncol Rep 29: 2019-2024, 2013.

34. Hwang MK, Kang NJ, Heo YS, Lee KW and Lee HJ: Fyn kinase is a direct molecular target of delphinidin for the inhibition of cyclooxygenase- 2 expression induced by tumor necrosis factor-alpha. Biochem Pharmacol 77: 1213-1222, 2009.

35. Duffy CP, Elliott CJ, O'Connor RA, et al: Enhancement of chemotherapeutic drug toxicity to human tumour cells in vitro by a subset of non-steroidal anti-inflammatory drugs (NSAIDs). Eur J Cancer 34: 1250-1259, 1998. 